\title{
Multi Input Sliding Mode Control of an Autonomous Fuel Cell-Supercapacitor Hybrid System
}

\author{
J.J. Moré*†, P.F. Puleston*, C. Kunusch** and E. Fossas***
}

\begin{abstract}
This paper presents the design of a sliding mode control strategy through an input decoupling of an hybrid fuel cell based system. The hybrid system is composed by a fuel cell stack and a Supercapacitors module, connected to a single DC bus. A continuous conduction mode MIMO model is considered and the control strategy for the system is discussed. The latter is developed using a diffeomorphism to decouple the control inputs to surface interactions. Finally, an adaptive switching function for the two inputs is designed to robustly reject system perturbations and parameters variations. The control strategy is then evaluated through extensive simulation, considering strong load demand variations.
\end{abstract}

\section{INTRODUCTION}

Fuel cell power systems have witnessed intense development in recent years as they offer a clean and efficient alternative for electrical power generation. Among them, Proton Exchange Membrane Fuel Cells (PEMFC) is a particularly promising technology. Producing electricity directly from the electrochemical oxidation of hydrogen. Additionally, their compactness, efficiency, and low operating temperature make them appropriate for a wide range of applications, from stationary to transportation and portable applications [1].

In this context, hybrid decentralised generation systems that incorporate renewable energy sources with a PEMFC module, are of special interest. Hydrogen can be easily produced, for instance, from renewable energies and it can be efficiently converted into electricity by means of fuel cells. The capability to store considerable amounts of energy through hydrogen gives more significance to such hybrid topologies, adding a great deal of flexibility. The core of this type of hybrid systems, comprises the aforementioned Fuel Cell Generation Module (FCGM) and a storage module (based on batteries or, most recently, supercapacitors), which is integrated to the FCGM, mitigating its exposure to fast loads [2] [3].

Properly controlled, these Fuel Cell based Hybrid Systems (FCBHS) present an excellent dynamic behaviour, high efficiency and non polluting emissions as the only byproducts are water and heat. However, they have serious operation problems and a great deal of multidisciplinary efforts, coming from electrochemistry, materials technology, fluid-dynamics, thermodynamics, automatic control and electronics, are indispensable to take up the challenge [4].

\footnotetext{
* CONICET and LEICI, Facultad de Ingeniería, Universidad Nacional de La Plata, Argentina

†jmore@ing.unlp.edu.ar

** Institut de Robótica i Informàtica industrial (CSIC-UPC), Parc Tecnològic de Barcelona. C/ Llorens i Artigas 4-6. 08028 Barcelona, Spain

*** IOC and Dept. of Automatic Control, Universitat Politècnica de Catalunya, Barcelona, Spain
}

The expected contribution from the control field is a set-up capable to ensure output voltage regulation, adequate power management and smooth fuel cell currents, even facing an exacting demand and the disturbances and uncertainties inherent to this type of systems. Over the past few years, significant work has taken place in the area.

For instance, in [2] supercapacitors were integrated to the system, improving significantly the power transient when PID controllers regulate the DC bus voltage via power converters. In [5] a hybrid system uses fuel cells for low frequency load requirements and the batteries in more demanding conditions, increasing the stack lifetime. In many applications, specific power converters have been designed to enhance the system efficiency and extend its operating range (e.g. voltage-clamping and resonant soft-switching [6]). Abridging, a wide range of linear control solutions that efficiently solve different control objectives have been proposed.

However, despite these and other recent advances in hybrid fuel cells based generation systems controllers, a considerable R\&D work is still necessary and a suitable comprehensive control approach, particularly able to cope with their challenging features, is needed. At this point, Sliding Mode Control (SMC) emerges as an especially apt technique to tackle the characteristics inherent to fuel cell systems. In addition, the on-line computational burden of the resultant algorithms can be considerably low, compared to those obtained with other non linear control techniques. Effectively, since its origins [7] [8], SMC has evolved into a robust and powerful design technique for a wide spectrum of applications, particularly nonlinear systems, MIMO plants and perturbed and uncertain systems [9] [10] [11].

In this paper a FCBHS combining a FCGM and Supercapacitors Bank (SCB) in a parallel connection is considered. This system topology is based on a real FCBHS test station intended for control assessment and evaluation. A MIMO SMC strategy is developed and designed through an input to surfaces decoupling transformation. The article is organized as follows:

Section II presents the system structure, its characteristics and model. In Section III the control strategy and the decoupled switching functions are described and developed. In Section IV, the simulation results are presented. Finally, in Section V the conclusions and future work is discussed.

\section{SYSTEM DESCRIPTION}

The core of the system under consideration consists of a FCGM connected to a DC bus through an unidirec- 
tional DC/DC power converter. The FCGM is based on a $1.2 \mathrm{~kW} \mathrm{Nexa}^{\circledR}$ Power Module from Ballard ${ }^{\circledR}$. The maximum current of this power module is $45 \mathrm{~A}$, while the output voltage ranges from around $24 V$ at rated current to about $45 \mathrm{~V}$ under open loop conditions. Its important to mention that the fuel cell stack is connected to the power converter through a low-pass filter, so the high switching frequency of the PWM signal do not affect the FC operation.

The FCBHS is completed by a second power channel based on a SCB connected to the same DC bus, in this case by a bidirectional DC/DC power converter. The SCB is intended to serve as an auxiliary power source of the hybrid system, and consists of a $165 F$ Maxewell ${ }^{\circledR}$ module. The rated voltage of the bank is $48 \mathrm{~V}$ and a maximum constant current of $98 \mathrm{~A}$.

The DC/DC power converters are based on two IGBT's columns of a Semistack ${ }^{\circledR}$ three-phase inverter from Semikron ${ }^{\circledR}$. The maximum current allowed through each switch of the columns is $75 \mathrm{Arms}$, and a maximum voltage of $750 \mathrm{~V}$. The maximum switching frequency of the IGBT's is $20 \mathrm{kHz}$. The nominal converters inductances are of $35 \mu \mathrm{Hy}$ and the DC bus capacitance has a value of $2720 \mu \mathrm{F}$.

In this case, the load is considered to be directly connected to the DC bus. A general scheme of the system described is shown in Fig. 1, where $P_{F C}, P_{S C}$ and $P_{0}$ stands for fuel cell, supercapacitors and load power flow, respectively.

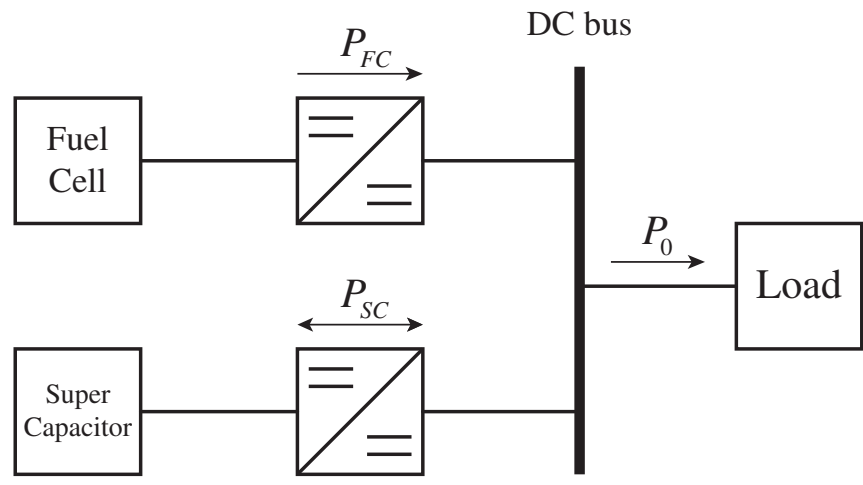

Fig. 1. Hybrid System Block Diagram

\section{A. System Model}

To obtain a dynamical model of the system, a schematic circuit diagram is presented in Fig. 2.

In this way, the following equations (1) to (4) represent an averaged model of the system dynamics, assuming continuous conduction mode and considering only the resistive losses on the inductances of the converters:

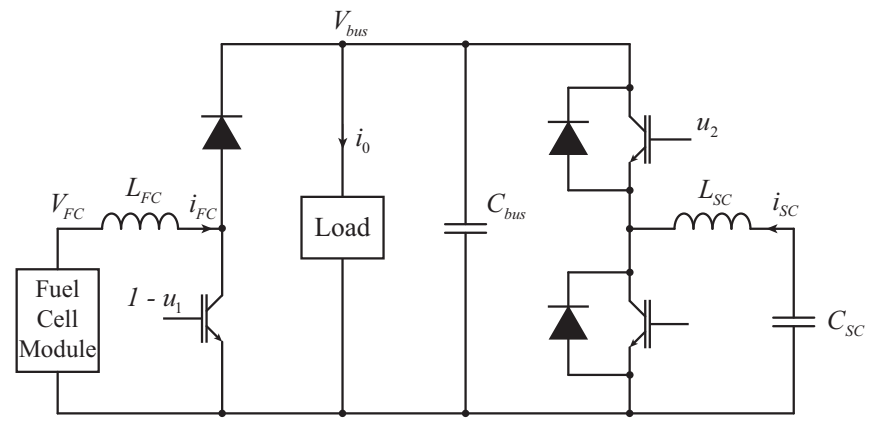

Fig. 2. Hybrid System Circuit Diagram

$$
\begin{aligned}
& \dot{i}_{F C}=\frac{v_{F C}}{L_{F C}}-\frac{R_{L f c} \cdot i_{F C}}{L_{F C}}-\frac{v_{b u s}}{L_{F C}} \cdot u_{1} \\
& \dot{i}_{S C}=\frac{v_{S C}}{L_{S C}}-\frac{R_{L s c} \cdot i_{S C}}{L_{S C}}-\frac{v_{b u s}}{L_{S C}} \cdot u_{2} \\
& \dot{v}_{b u s}=-\frac{i_{0}}{C_{b u s}}+\frac{i_{F C}}{C_{b u s}} \cdot u_{1}+\frac{i_{S C}}{C_{b u s}} \cdot u_{2} \\
& \dot{v}_{S C}=-\frac{i_{S C}}{C_{S C}}
\end{aligned}
$$

where:

$$
\begin{aligned}
v_{F C}= & N_{F C} \cdot\left(E_{o c}-R_{F C} \cdot i_{F C}-\right. \\
& \left.-A \cdot \log \left(i_{F C}\right)-m \cdot \exp \left(n \cdot i_{F C}\right)\right)
\end{aligned}
$$

represents the output voltage of the FC as a current function, with:

$\begin{array}{ll}i_{F C}, i_{S C} & \begin{array}{l}\text { the FCGM and SCM current; } \\ v_{b u s}, v_{S C}\end{array} \\ C_{b u s}, C_{S C} \text { the DC bus and SCM voltage; } & \text { the DC bus and SCM capacitance; } \\ L_{F C}, L_{S C} & \text { the FCGM and SCM converter inductances; } \\ i_{0} & \text { the load current; } \\ u_{i} & \text { the duty cycle of the upper column switch } \\ & \text { PWM control signal } i ; \\ R_{L f c} & \text { the FC converter inductor resistance; } \\ R_{L s c} & \text { the SCM converter inductor resistance; } \\ N_{F C} & \text { the number of cells of the FC stack; } \\ E_{o c} & \text { the open loop voltage of a cell; } \\ R_{F C} & \text { the linear resistance of the FC; } \\ A & \text { the slope of the Tafel equation; } \\ m, n & \text { constants of the mass transfer overvoltage }\end{array}$

A classical system model structure is presented here, that would be used in the next section:

$$
\dot{x}=f(x)+g(x) \cdot u
$$

where $x=\left[\begin{array}{llll}i_{F C} & i_{S C} & v_{\text {bus }} & v_{S C}\end{array}\right]^{T}$ and $u=\left[\begin{array}{ll}u_{1} & u_{2}\end{array}\right]^{T}$ are the system states and inputs vectors respectively, and:

$$
f(x)=\left[\begin{array}{c}
\frac{v_{F C}}{L_{F C}} \\
\frac{v_{S C}}{L_{S C}} \\
-\frac{i_{0}}{C_{b u s}} \\
-\frac{i_{S C}}{C_{S C}}
\end{array}\right]
$$




$$
g(x)=\left[\begin{array}{cc}
-\frac{v_{b u s}}{L_{F C}} & 0 \\
0 & -\frac{v_{b u s}}{L_{S C}} \\
\frac{i_{F C}}{C_{b u s}} & \frac{i_{S C}}{C_{b u s}} \\
0 & 0
\end{array}\right]
$$

\section{CONTROL DESIGN}

\section{A. Control Strategy}

The main objective of the control algorithm is to guarantee a constant DC bus voltage and that the load is supplied with the required power, even under strong load variations. To achieve this objective, it must be considered that both the value and rate of change of the FCGM current must be carefully limited under normal operations. The stack current range should be fixed in order to ensure that the FCM is working within the linear region of its polarization curves. Moreover, under variable load conditions, the stack current slope should be limited to protect the device and extend its durability. Two main reasons can be stated for that. Firstly, the time constant of the stack voltage response limits the power delivered to the load, i.e. FC's power cannot be set arbitrarily fast. Secondly, fast current changes may produce thermal stress at the catalyst surface, decreasing the membrane lifetime.

In this way, the SCM must complement the FCGM power to accurately supply the load. This requires that the SCM converter must act rapidly to reject abrupt load variations. After the transient conditions due to load variations, the FCGM must slowly recharge the SCM bank to a desired value, avoiding to overload the stack.

To summarize, a MIMO sliding mode control strategy that fulfil the above mentioned restrictions and objectives is designed in the next subsections. The control objectives are covered by suitable surfaces and current references selection.

\section{B. Fuel Cell Converter Sliding Surface}

The FCGM converter sliding surface is designed to follow smooth load power variations, considering the FCM current limitations mentioned in the previous subsection. In stationary state, the FCM must supply the load power or its maximum rated value. In addition, the FCM is responsible of recharging the SCM bank to a desired value. The above mentioned requirements are fulfilled by the following sliding surface structure:

$$
\begin{aligned}
s_{1} & =i_{F C}-I_{\text {TotalFCref }} \\
& =i_{F C}-I_{F C r e f}+a_{1} \cdot\left(v_{S C}-V_{S C r e f}\right)
\end{aligned}
$$

where:

$I_{\text {TotalFCref }}$ a lumped FC current reference. This current reference has a slope limitation to a safe value in order to assure an accurate behavior of the FCM. It is also important to mention that it is also limited to a maximum value of $45 \mathrm{~A}$, the maximum allowed current for the FC system.

$V_{S C r e f} \quad$ SCM voltage reference;

$a_{1} \quad$ positive design gain of the sliding surface. This gain must be selected considering the desired SCM bank charging dynamics. Small values for $a_{1}$ result in a slow recharging dynamics, while higher values imply faster SCM charging times, but may overload the FCGM;

$I_{F C r e f} \quad$ is the current that the $\mathrm{FC}$ has to deliver to supply the required power to the load. This current reference is calculated as follows:

$$
I_{F C r e f}=\frac{P_{0}}{v_{F C}}
$$

where $P_{0}=v_{b u s} \cdot i_{0}$ is the load power.

\section{Supercapacitor Converter Sliding Surface}

The SCM converter sliding surface takes into account the slow response of the FCM to follow load power variations. In this way, the SCM must reject the high frequency load changes and supply the lack of FCM power. Thus, tacking advantage of the fast dynamic response of the SCM, the sliding surface is designed to regulate the DC bus voltage. In this case, a classical boost output voltage regulation surface is considered, combining both bus voltage and SCM current:

$$
s_{2}=i_{S C}-I_{S C r e f}+a_{2} \cdot\left(v_{b u s}-V_{\text {busref }}\right)
$$

where:

$V_{\text {busref }}$ is the bus voltage reference;

$a_{2} \quad$ a positive surface gain selected to obtain a desired close loop $v_{\text {bus }}$ dynamics.

$I_{S C r e f}$ is obtained based on the power difference between the FC and load power demand, i.e.:

$$
I_{S C r e f}=\frac{P_{0}-P_{F C}}{v_{S C}}
$$

with $P_{F C}=v_{F C} \cdot i_{F C}$ the actual power that FC module delivers to the DC bus.

\section{Decoupled Control Action Design}

To design the control inputs and switching conditions, an input to surface decoupling diffeomorphism is considered. Defining vector $S=\left[\begin{array}{ll}s_{1} & s_{2}\end{array}\right]^{T}$ and calculating the sliding variables time derivative:

$$
\begin{aligned}
\dot{S} & =\frac{\partial S}{\partial x} \cdot \dot{x}+\frac{\partial S}{\partial t} \\
& =\frac{\partial S}{\partial x} \cdot f(x)+\frac{\partial S}{\partial x} \cdot g(x) \cdot u+\frac{\partial S}{\partial t}
\end{aligned}
$$

Let now consider a diffeomorphism $T(x)$ and a new control input vector $w$, so:

$$
w=T(x) \cdot u
$$

Selecting $T(x)=\frac{\partial S}{\partial x} \cdot g(x)$ [12], equation (14) becomes:

$$
\begin{aligned}
\dot{S} & =\frac{\partial S}{\partial x} \cdot f(x)+\frac{\partial S}{\partial x} \cdot g(x) \cdot T(x)^{-1} \cdot w+\frac{\partial S}{\partial t} \\
& =\frac{\partial S}{\partial x} \cdot f(x)+I \cdot w+\frac{\partial S}{\partial t}
\end{aligned}
$$


where $I$ is the identity matrix. In this way, the control input $w_{i}$ acts over the surface $s_{i}$, and both of the control actions can be designed independently. The matrix $T(x)$ results:

$$
T(x)=\left[\begin{array}{cc}
-\frac{v_{b u s}}{L_{F C}} & 0 \\
\frac{a_{2} \cdot i_{F C}}{C_{b u s}} & \frac{a_{2} \cdot i_{S C}}{C_{b u s}}-\frac{v_{b u s}}{L_{S C}}
\end{array}\right]
$$

The inverse of this matrix always exists if $v_{b u s} \neq 0$ and $\frac{a_{2} \cdot i_{S C}}{C_{b u s}}-\frac{v_{b u s}}{L_{S C}} \neq 0$. The former condition is always true given that the FCM always provides a positive voltage at the FC converter input. The latter condition can be achieved by tacking into account the operation range and designing the surface parameter $a_{2}$ accordingly.

With the previous considerations, taking $\dot{S}=0$ and $S=0$, the nominal equivalent control action is obtained:

$$
\begin{aligned}
& w_{e q N}=-\frac{\partial S}{\partial t}-\left.\frac{\partial S}{\partial x} \cdot f(x)\right|_{S=0} \\
& =\left[\begin{array}{c}
-\frac{v_{F C}}{L_{F C}}+\frac{R_{L f c} \cdot i_{F C}}{L_{F C}}+\frac{a_{1} \cdot i_{S C}}{C_{b u s}}+\dot{I}_{F C r e f} \\
-\frac{v_{S C}}{L_{S C}}+\frac{R_{L s c} \cdot i_{S C}}{L_{S C}}+\frac{a_{2} \cdot i_{0}}{C_{b u s}}+\dot{I}_{S C r e f}
\end{array}\right]
\end{aligned}
$$

and

$$
\left.\left[\begin{array}{c}
i_{F C} \\
i_{S C}
\end{array}\right]\right|_{S=0}=\left[\begin{array}{c}
I_{F C r e f}-a_{1} \cdot\left(v_{S C}-V_{S C r e f}\right) \\
I_{S C r e f}-a_{2} \cdot\left(v_{\text {bus }}-V_{\text {busref }}\right)
\end{array}\right]
$$

Its important to mention that $\dot{I}_{F C r e f}$ and $\dot{I}_{S C r e f}$ in equation (18) are obtained from filtered signals of $I_{F C r e f}$ and $I_{S C r e f}$, respectively. This is significant to avoid noise amplification and high peeks due to fast changes in the SC current reference.

The adaptive control law applied to the system is selected to be of the form:

$$
w_{i}=w_{e q N i}+\left(W_{c i}+W_{a i} \cdot\left|i_{i}\right|\right) \cdot \operatorname{sign}\left(s_{i}\right)
$$

where

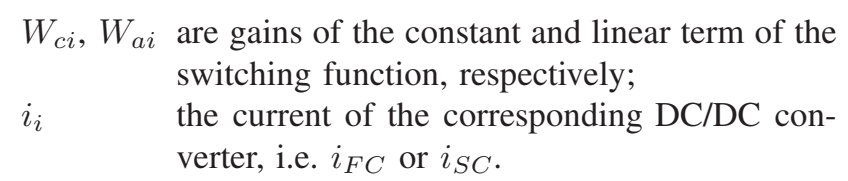

The first term of (20) maintains the nominal system over the surface. The second term, allows to robustly reject parameter variations, miscalculations, unmodeled dynamics and perturbations. Its important to emphasize that the main effects of unmodeled losses of the converters are directly associated to the current. In this way, the linear term of the discontinuous function in (20) is added, to mitigate its impact. Thus, this current adaptive discontinuous control term in (20) can be reduced if the unmodeled converters losses are reduced. Then, chattering effects can be significantly diminished.

If necessary, in practical implementations an integral term can be incorporated into the references to reject miscalculations. Furthermore, a term $w_{\text {reach }}$ may be added to (20) to ensure a fast reaching dynamics, i.e.:

$$
w_{\text {reach }}= \begin{cases}\left|s_{i}\right| \cdot \operatorname{sign}\left(s_{i}\right) & \left|s_{i}\right|>\epsilon \\ 0 & \left|s_{i}\right|<\epsilon\end{cases}
$$

with $\epsilon$ a given positive constant.

It must be mentioned that in this application, the system operates under continuous conduction mode and the control design is based on this fact. However, the authors are working to extend the present control strategy to applications involving both continuous and discontinuous conduction modes.

\section{SIMULATION RESULTS}

This section presents the simulations results of the control strategy designed, under strong power demand and converters parameter variations. The switching functions gains $W_{c i}$ and $W_{a i}$ were designed tacking into account $10 \%$ system parameters variations. Nominal values for $R_{L f c}$ and $R_{L s c}$ of $30 \mathrm{~m} \Omega$ were considered. On the other hand, the parameters of equation (5) were taken as follows, to fit the $N e x a^{\circledR}$ polarization curve:

$$
\begin{array}{ccc}
N_{F C}=47 ; & E_{\text {oc }}=1.031 ; & R_{F C}=2.45 \times 10^{-4} ; \\
A=0.03 ; & m=2.11 \times 10^{-5} ; & n=0.008 ;
\end{array}
$$

As a main system perturbation, strong power demand variations are simulated. In particular, step-like transitions of the load demand are considered, forcing the power and current slope limitation to act. The surface gain $a_{1}$ was set to 1 , obtaining an adequately SCM charging dynamics. In turn, the surface gain $a_{2}$ was configured to 20 , to rapidly reject load power perturbations. The DC bus voltage reference was set to $75 \mathrm{~A}$ and de SCM voltage reference to $40 \mathrm{~V}$, respectively. The switching frequency was limited to $20 \mathrm{kHz}$, considering the maximum frequency allowed by the DC/DC converters. The actual power demand of the load is depicted in Fig. 3. Note that, from 35 to $40 \mathrm{sec}$ a power demand that exceeds the FC module rated power, enabling the FCM maximum current limitation to act.

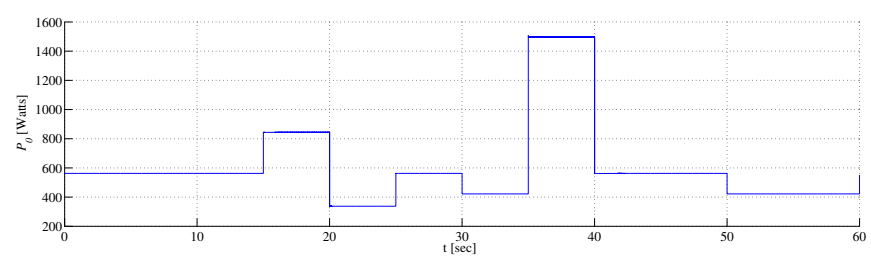

Fig. 3. Load Power Demand $P_{0}$

In Fig. 4 and Fig. 5 the DC bus voltage $v_{b u s}$ and current $i_{0}$ are shown. In spite of the fact that exists abrupt variations of the load demand, it can be seen that the control strategy regulates de DC bus voltage to the reference value. This is achieved also under a power step of $100 \%$ of installed power at time $35 \mathrm{sec}$.

Figures 6 and 7 depict the FCM voltage and current, respectively. In this case, the slope limitation of the current variation can be observed. The FC maximum current limitation acts around time $37 \mathrm{sec}$, by accurately restricting its reference value.

Fig. 8 shows de SCM module voltage. In this case, when the power demand is constant, the SCM slowly recharges to its reference value. On the other hand, in transient conditions, 


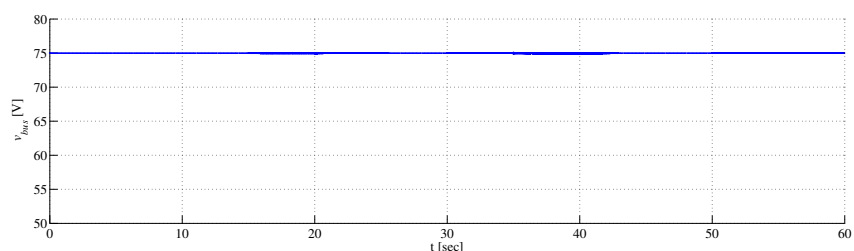

Fig. 4. DC bus Voltage $v_{\text {bus }}$

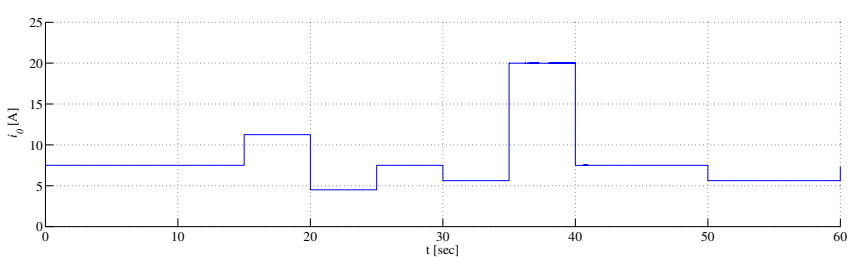

Fig. 5. Load Current $i_{0}$

the SCM charges or discharges as required (Fig. 9). From the current of the SCM converter, a fast response to the load variation is appreciated. At time $35 \mathrm{sec}$, when load demand is higher than the FC rated power, the SCM module still regulates the DC bus voltage by keeping in discharge mode, as seen in Fig. 9. In this case, this condition can be ensured only for a reduced period of time, as the SCM will discharge to zero or a minimum function voltage.

Figures 10 and 11 depict the duty cycles applied to the converters, i.e. $u=T(x)^{-1} \cdot w$. Finally, Figures 12 and 13 show the surfaces. From Fig. 12, associated to the FC module converter, it can be concluded that the system always stays over the surface. On the other hand, the adaptive switching signal designed can be appreciated from this figure, as the chattering effect is higher as the converter current is also higher.

In Fig. 13, is shown that the system is maintained over the $s_{2}$ surface, except when the load demand abruptly changes. This disengagement occurs mainly due to the filter used to calculate the $I_{S C r e f}$ derivative, as mentioned in the subsec-

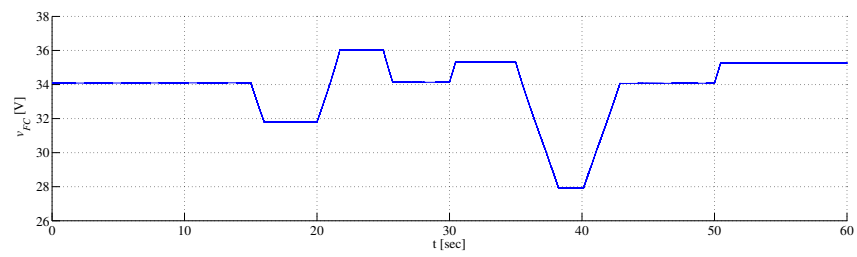

Fig. 6. Fuel Cell Votege $v_{F C}$

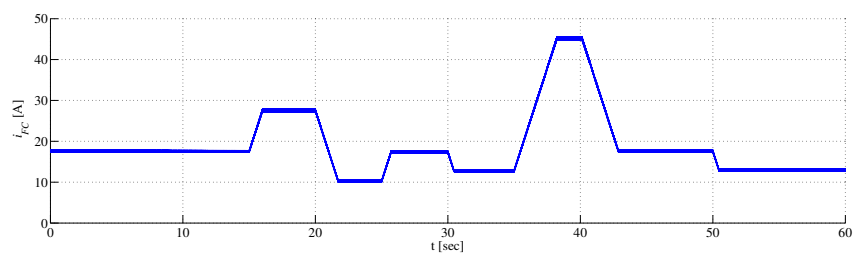

Fig. 7. Fuel Cell Current $i_{F C}$

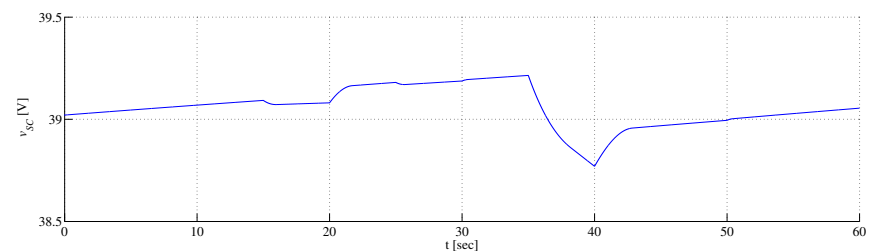

Fig. 8. Supercapacitor Voltage $v_{S C}$

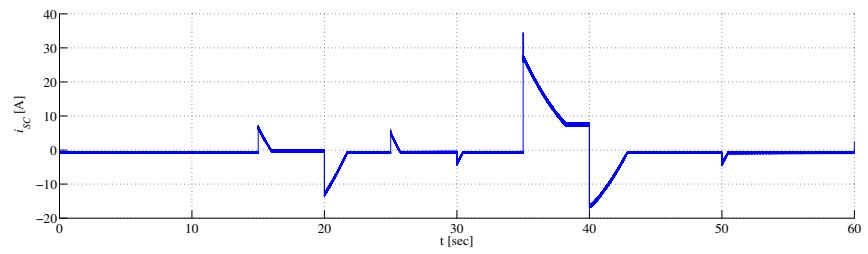

Fig. 9. Supercapacitor Current $i_{S C}$

tion III-D. However, the control strategy rapidly reaches the surface again, rejecting the perturbation.

\section{CONCLUSIONS}

In this paper, a MIMO sliding mode control of a fuel cell/supercapacitor based hybrid system was presented. The hybrid system consisted of a fuel cell module connected through a unidirectional DC/DC power converter to a DC bus and a supercapacitors bank coupled to the same DC bus via a bidirectional power converter.

The general control strategy was based in the fact that the fuel cell current should not be changed rapidly, to avoid possible irreversible damage to the stack. In this way, the supercapacitors bank module needs to complement the fuel cell power during transient variations of load demand. After these transient events, the fuel cell module should meet the load demand and slowly recharge the supercapacitors bank to a desired value. In addition, this process must be done while maintaining the DC bus voltage at a constant reference value.

To this end, two sliding surfaces were considered in order to meet the specifications. The sliding control law for both

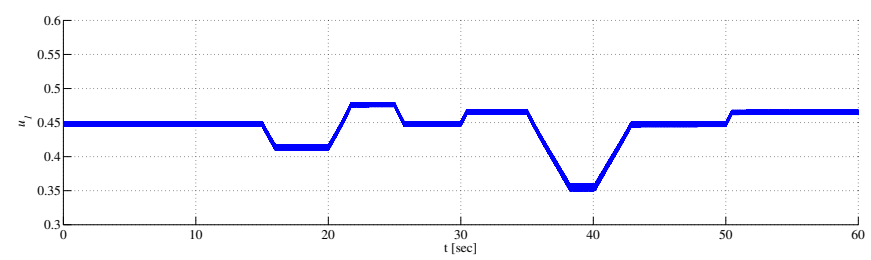

Fig. 10. Fuel Cell Converter Duty Cycle $u_{1}$

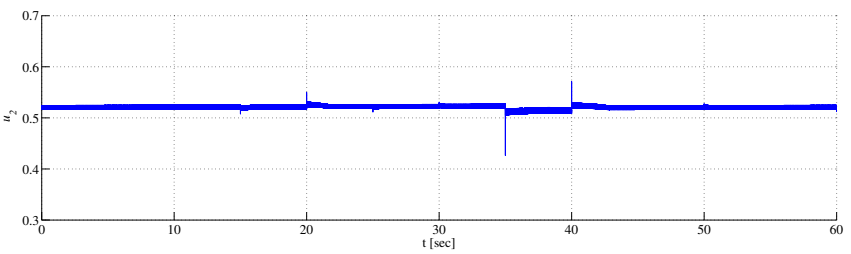

Fig. 11. Supercapacitor Converter Duty Cycle $u_{2}$ 


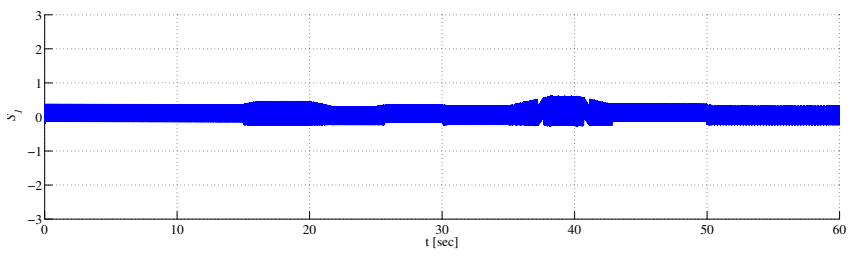

Fig. 12. Fuel Cell Converter Surface $S_{1}$

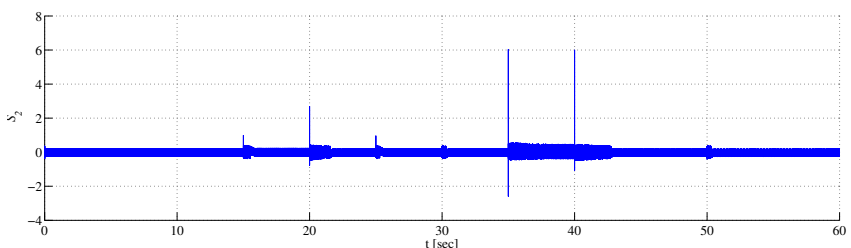

Fig. 13. Supercapacitor Converter Surface $S_{2}$

surfaces was designed applying a input to surface decoupling diffeomorphism. Finally, an adaptive control law was applied to robustly reject system perturbations and model uncertainties.

The closed loop performance has been thoroughly assessed through extensive simulation tests, including several load disturbances and parameter uncertainties. The results were extremely satisfactory. The dynamic response to sudden load variations proved to be fast, showing a minimum DC bus voltage drop, even in the presence of demand steps of $100 \%$ of installed power. Moreover, the control strategy prove to gradually restore the supercapacitor voltage to the reference value, avoiding to overload the fuel cell. Furthermore, the closed loop system is also able to meet load requirements higher than the fuel cell rated power, in this case for short time periods.

The next step in this research project is the implementation and experimental validation in the laboratory hybrid test station of the proposed strategy and control laws. Its also important to emphasize that a complete hybrid autonomous system is under study, adding another renewable energy source and an electrolyzer. In particular, a suitable source to this specific case is a wind energy conversion system. Moreover, the design and implementation of a supervisory control strategy of the entire hybrid system is been developed.

\section{ACKNOWLEDGMENTS}

This research was supported by the Universidad Nacional de La Plata, the Consejo Nacional de Investigaciones Científicas y Técnicas (CONICET), and SECyT, from Argentina; and by the 7FP of EU under project ACOFC (GA293876); and by the Universitat Politècnica de Catalunya, the MICINN under CICYT projects DPI2010-15274 and DPI2010-15110, the CSIC under JAEDOC Research Programme and the AECID under project A/026279/09, from Spain.

\section{REFERENCES}

[1] F. Barbir, PEM fuel cells: theory and practice. Elsevier, 2005.

[2] P. Thounthong, S. Raël, and B. Davat, "Control strategy of fuel cell/supercapacitors hybrid power sources for electric vehicle," Journal of Power Sources, vol. 158, p. 806-814, 2006.

[3] — "Control strategy of fuel cell and supercapacitors association for a distributed generation system," IEEE Transactions on Industrial Electronics, vol. 54, p. 3225-3233, 2007.

[4] C. Kunusch, P. Puleston, M. Mayosky, and J. Husar, "Control-oriented modeling and experimental validation of a PEMFC generation system," IEEE Transactions on Energy Conversion, vol. 26, no. 3, pp. 851-861, 2011.

[5] H.-S. Lee, K.-S. Jeong, and B.-S. Oh, "An experimental study of controlling strategies and drive forces for hydrogen fuel cell hybrid vehicles," International Journal of Hydrogen Energy, vol. 28, pp. 215222, 2003.

[6] R.-J. Wai, R.-Y. Duan, J.-D. Lee, and L.-W. Liu, "High-efficiency fuel-cell power inverter with soft-switching resonant technique," IEEE Transactions on Energy Conversion, vol. 20, pp. 482-492, 2005.

[7] S. Emelyanov, Variable Structure Control Systems, Moscow, Nauka., 1967.

[8] V. Utkin, "Variable structure systems with sliding modes," IEEE Transactions on Automatic Control, vol. 22, no. 2, pp. 212-222, 1977.

[9] A. Sabanovic, L. M. Fridman, and S. Spurgeon, Variable Structure Systems: from Principles to Implementation. IEE, 2004.

[10] C. Edwards, E. Fossas Colet, and L. Fridman, Advances in Variable Structure and Sliding Mode Control. Springer, 2006, vol. 334.

[11] B. Bartolini, L. Fridman, A. Pisano, and L. Usai, Modern Sliding Mode Control Theory: New Perspectives and Applications, B. Bartolini, L. Fridman, A. Pisano, and L. Usai, Eds. Springer, 2008.

[12] H. Sira-Ramirez, "Differential geometric methods in variable structure control," International Journal of Control, vol. 48, no. 5, pp. 13591390, 1988. 